\title{
Tomato Juice Consumption Could Improve Breast Skin Adverse Effects of Radiotherapy in Breast Cancer Patients
}

\author{
YASUYO FUKUSHI ${ }^{1,2}$, YASUSHI MARIYA ${ }^{2,3}$, KYOGO YAMADA $^{4}$, KAZUE YOSHIDA $^{2}$, \\ ASAMI SASA ${ }^{2}$, HITOSHI SAITO ${ }^{5}$, AYUMI HIRAI ${ }^{6}$, SHIGENORI SUZUKI ${ }^{6}$, \\ KOICHI AIZAWA ${ }^{6}$, HIROYUKI SUGANUMA ${ }^{6}$ and CHIEKO ITAKI ${ }^{7}$ \\ ${ }^{1}$ Department of Disability and Health, Division of Health Sciences, \\ Hirosaki University Graduate School of Health Sciences, Hirosaki, Japan; \\ ${ }^{2}$ Department of Radiology, Mutsu General Hospital, Mutsu, Japan; \\ ${ }^{3}$ Department of Radiation Oncology, Aomori Rosai Hospital, Hachinohe, Japan; \\ ${ }^{4}$ Department of Surgery, Mutsu General Hospital, Mutsu, Japan; \\ ${ }^{5}$ Department of Central laboratory, Mutsu General Hospital, Mutsu, Japan; \\ ${ }^{6}$ Innovation Division, Kagome Co., Ltd., Nasushiobara, Japan; \\ ${ }^{7}$ Department of Nursing Science, Hirosaki University Graduate School of Health Sciences, Hirosaki, Japan
}

\begin{abstract}
Background/Aim: We investigated the beneficial effects of drinking tomato juice (TJ) rich in antioxidant carotenoids on irradiated skin following radiotherapy $(R T)$ in breast cancer patients. Patients/Methods: Twenty-three patients agreed to drink TJ (160 g/day for six months) after the completion of RT. Early and late adverse events (AEs) of irradiated skin were evaluated according to the Common Terminology Criteria for AEs and the European Organization for Research and Treatment of Cancer Global Cosmetic Rating System, respectively. Results: With regard to early AEs, acute radiodermatitis of grade 1 was observed in most patients (22/23) at the end of RT. However, the grade of radiodermatitis rapidly changed to 0,1 month after $R T$ and starting TJ consumption. With regard to late AEs, most patients were in good or excellent dermal condition. Conclusion: TJ consumption could help in relieving and recovering from early AEs and decreasing the severity of late AEs of irradiated skin.
\end{abstract}

The living body is exposed to radiation in cancer radiotherapy to target and damage cancer cells. There are

This article is freely accessible online.

Correspondence to: Chieko Itaki, Department of Nursing Science, Hirosaki University Graduate School of Health Sciences, 66-1 Honcho, Hirosaki City, Aomori 036-8564, Japan. Tel: +81 172395974, Fax: +81 172395974, e-mail: itakichi@hirosaki-u.ac.jp

Key Words: Tomato juice, carotenoids, lycopene, radiotherapy, breast cancer. two known routes to express the effects of radiotherapy (1). One is the direct action of radiation directly damaging the target molecules, and the other is the indirect action. The effectiveness is $30 \%$ for the former and $70 \%$ for the latter (1). In the indirect action, radiation first acts on water, which makes up about $60-80 \%$ of the body producing reactive oxygen species (ROS) that damage DNA (1). Oxidative stress represented by ROS continuously occurs under physiological conditions. However, ROS can also be caused by factors such as smoking, strenuous exercise, radiation, ultraviolet light and air pollutants. In vivo, ROS has the role of removing foreign substances such as bacteria in addition to being associated with an antitumor effect (2). On the other hand, when ROS is produced in excess, it is known to react with biopolymers such as DNA, lipids and proteins and cause dysfunction and canceration of normal cells in the living body $(2,3)$. Therefore, a mechanism to efficiently eliminate ROS is also important. In vivo, an antioxidant network exists to protect healthy tissues from oxidative stress (4). Antioxidants, such as vitamin C, vitamin E, and carotenoids, one of the antioxidant networks, scavenges free radicals. Ingestion of antioxidants that constitute the antioxidant network into the body is believed to have a positive effect on the oxidative stress state in vivo. Recently, carotenoids in particular, have attracted attention among antioxidants (2). Carotenoids have been reported to be associated with the reduction in the risk of chronic diseases such as cardiovascular disease, metabolic syndrome and cancer (2). Proactive intake of carotenoids is believed to have beneficial effects on health. Among carotenoids, lycopene has a particularly potent ability to eliminate singlet oxygen, which is a type of ROS. The potency is said to be 
twice that of $\beta$-carotene and about 100 times that of $\alpha$ tocopherol (3).

Previously, we requested ten healthy people to drink tomato juice, which is rich in carotenoids, daily for a specific period. We reported that increased carotenoids in human plasma could reduce damage to DNA caused by radiation to human lymphocytes (5). In this study, we focused on the relationship between consumption of tomato juice and adverse events of skin caused by radiotherapy (6). Among the adverse events of skin, radiodermatitis, an adverse event that occurs at an early stage, during or shortly after the completion of radiotherapy, shows symptoms such as erythema, warmth and dryness on the skin in the irradiation field, as the patients gradually recover from the symptoms. Adverse events occurring at the late stage ranging from six months to a few years following irradiation after the adverse events of the early stage, include symptoms such as fibrosis of the skin, pigmentation, and continued dryness, due to decreased secretion of sweat and sebaceous glands, in the irradiation field. If consumption of tomato juice contributes to the prevention of deterioration and promotes recovery of skin condition by improving oxidative stress, it is expected to contribute to maintaining the quality of life (QOL) and early improvement of cancer patients receiving radiotherapy. In particular, the significance of breast conservation therapy is high in the case of breast cancer as the absolute number of patients is high, and breast shape after treatment is important. Up to now, there are few studies linking lycopene and antioxidants to reducing cancer risk (3) and consumption of tomato juice to oxidative stress levels (7). However, as far as we know, research on the protection by carotenoids or lycopene from radiation is still limited $(3,5)$. Also, studies on the clinical course of adverse events of the skin associated with the drinking of tomato juice and radiotherapy have not been reported in the past.

The purpose of this study was to examine in detail the effect of consuming tomato juice on the skin condition in the irradiation field after radiotherapy and its clinical usefulness in breast cancer patients following breast-conserving surgery.

\section{Patients and Methods}

Subjects and radiotherapy. We requested 23 patients with breast cancer who received postoperative radiotherapy after breastconserving surgery between October 2013 and March 2017, to drink tomato juice (TJ). The subjects had to drink tomato juice for six months from the next day following the end date of radiotherapy. We explained the consumption method of TJ to the patients and that TJ will be supplied for six months and obtained informed consent from the subjects before this study. Twenty-three subjects who agreed to consume TJ were included in this study. We continued to monitor the skin condition of the subjects up to 12 months after the end of radiotherapy. The subjects were between 29-84 (median 59) years of age. Radiotherapy was performed using the linear
Table I. Patient characteristics.

\begin{tabular}{llr}
\hline & & $(\mathrm{n}=23)$ \\
\hline Stage & 0 & 7 \\
& I & 6 \\
& IA & 0 \\
& II & 0 \\
& IIA & 9 \\
& IIB & 1 \\
& IIIA & 0 \\
Intrinsic subtype & IV & 0 \\
& Luminal A & 7 \\
& Luminal B & 10 \\
& TNBC & 4 \\
Performance status & HER-2 & 1 \\
Combination therapy & Undefined & 1 \\
& Chemotherapy & 23 \\
& Hormonal therapy & 1 \\
& Chemotherapy+Hormonal therapy & 5 \\
\hline
\end{tabular}

accelerator (CLINAC-iX, Varian Medical Systems, CA, USA) with tangential irradiation with $4 \mathrm{MV}$ or $4 \mathrm{MV}$ and $10 \mathrm{MV}$ X-rays. A conventional fractionation ( $2 \mathrm{~Gy} /$ fraction, five times/week) was used for a total target dose of $50 \mathrm{~Gy} / 25$ fractions. The patient characteristics are shown in Table I.

Consumption of TJ. We provided the subjects with commercially available TJ (lycopene content in one can: $16 \mathrm{mg} / 160 \mathrm{~g}$, no salt added) and as a general rule, we requested them to consume one can of TJ a day. The reason for selecting TJ is that it has high lycopene content and can be stored at room temperature. A fixed amount of lycopene is expected to be taken in every day if it is consumed as instructed. Nutritional contents per $160 \mathrm{~g}$ can of TJ used in this study include: energy $32 \mathrm{kcal}$, protein $1.3 \mathrm{~g}$, carbohydrates $6.1 \mathrm{~g}$, dietary fiber $1.1 \mathrm{~g}$, sodium 1-18 mg, calcium $11 \mathrm{mg}$ and potassium $460 \mathrm{mg}$. Concentration (analytical values) of the carotenoid components per can of TJ is as following: lutein $0.11 \pm 0.0009 \mathrm{mg}, \beta$-cryptoxanthin $0.03 \pm 0.0007 \mathrm{mg}$, $\alpha$-carotene $0.11 \pm 0.0009 \mathrm{mg}, \beta$-carotene $0.29 \pm 0.0028 \mathrm{mg}$ and lycopene $19.34 \pm 0.541 \mathrm{mg}$.

Data collection. Blood and urine were collected before or at the start of radiotherapy (Baseline), the week in which the treatment was ended (End), 1 month (1M), 3 months $(3 \mathrm{M})$ and 6 months $(6 \mathrm{M})$ after the end of radiotherapy. The skin condition of subjects was confirmed by multiple clinicians at Baseline, second week of radiotherapy $(2 \mathrm{w})$, third week of radiotherapy $(3 \mathrm{w})$, fourth week of radiotherapy ( $4 \mathrm{w})$, End, $1 \mathrm{M}, 3 \mathrm{M}, 6 \mathrm{M}$, and 12 months (12M) after the end of radiotherapy.

Measurement of serum carotenoid concentration. Serum carotenoid concentration was measured according to a previous report using highperformance liquid chromatography (HPLC) (8). The differences with the previous report were that $20 \mathrm{ul}$ of the internal standard substance was used, the mixed solution was stirred for $30 \mathrm{~s}$, centrifugation was performed at $1,710 \times \mathrm{g}$ for $5 \mathrm{~min}$ to obtain the supernatant, and a 0.22 um filter was used to filter the solution for HPLC. 


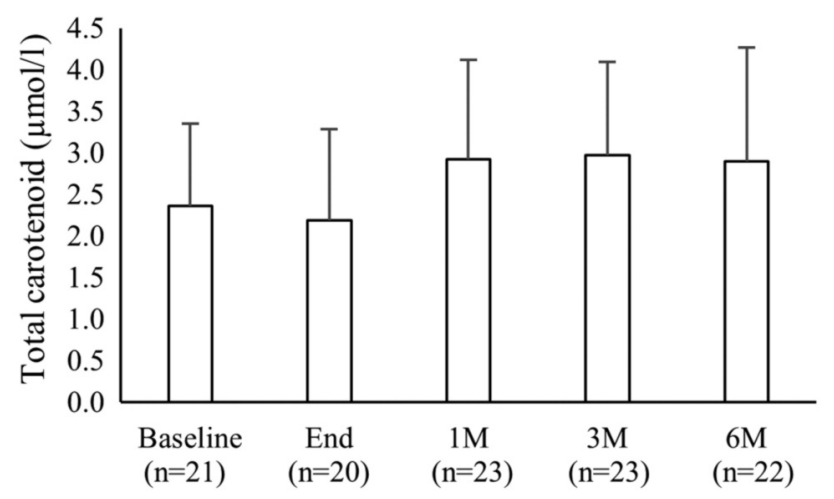

Figure 1. Serial change in total carotenoid concentration. After starting tomato juice consumption from the End timepoint to $6 \mathrm{M}$, concentration of total carotenoids increased. However, there was no significant difference in the values. The total carotenoid concentration data were compared by Kruskal-Wallis test. Mann-Whitney U-test was used as post hoc analysis to find significant differences, and p-values obtained were corrected by the Shaffer method. Columns and bars show average and standard deviation, respectively.

Measurement of skin condition. The skin condition was measured in an outpatient clinic, with the room temperature adjusted to $24-25^{\circ} \mathrm{C}$ using an air conditioner. As objective and quantitative markers, skin surface temperature and skin moisture were measured. Radiodermatitis, an early stage adverse event caused due to radiotherapy, was evaluated based on the Common Terminology Criteria for Adverse Events (CTCAE) v4.0 (9). The late adverse events 6 months after the end of radiotherapy were evaluated based on the scoring system of the Global Cosmetic Rating System of the European Organization for Research and Treatment of Cancer (EORTC) (10).

Measurement of skin surface temperature and skin moisture. Previous studies have reported changes in skin surface temperature and skin moisture for breast cancer patients undergoing radiotherapy (11). The measurement of skin surface temperature is particularly useful as a method for evaluating the skin condition. The measurement location selected was at the field of the breast that was irradiated, avoiding surgical wounds and markings. As a control, a measurement location was selected in the non-irradiation field that was symmetrical to the measurement location in the irradiation field. A non-contact infrared thermometer (Visiofocus Pro, Tecnimed Srl, Vedano Olona, VA, Lombard, Italy) was used to measure the skin surface temperature. The irradiation side and nonirradiation side were each measured twice, and the average of the measured values was defined as the skin surface temperature. Skin moisture was measured using the Mobile Moisture HP10-N instrument (Courage+Khazaka, Cologne, North Rhine-Westphalia, Germany). The irradiation side and non-irradiation side were each measured 5 times, and the highest and lowest values of the 5 measurements were excluded. The average of the remaining 3 measured values was defined as the value of skin moisture. Due to the significant individual differences in the measurement items, the value obtained by subtracting the value of the non-irradiation side from the value of the irradiation side was used for comparison in all evaluations. The difference in skin surface temperature was defined as dTemp (difference of skin surface temperature between

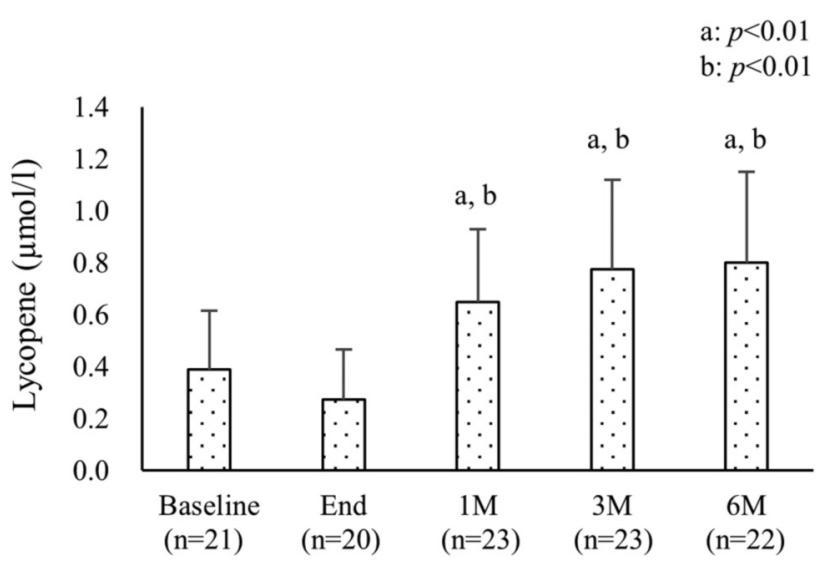

Figure 2. Serial change in lycopene concentration. After starting tomato juice consumption from End to $6 \mathrm{M}$, concentration of lycopene significantly increased. The lycopene concentration data were compared by the Kruskal-Wallis test. Mann-Whitney U-test was used as post hoc analysis to find significant differences, and p-values obtained were corrected by the Shaffer method. Column and bar show average and standard deviation, respectively. a: Significantly different from Baseline $(p<0.01)$. $b$ : Significantly different from End $(p<0.01)$.

ipsilateral and contralateral breasts), and the difference in skin moisture was defined as dMoist (difference of skin moisture between ipsilateral and contralateral breasts).

Judgement of CTCAE. Skin with slight erythema or dry desquamation in the irradiation field was judged as Grade 1, and skin with a milder condition was judged as Grade 0 .

Judgement of EORTC. Based on a comprehensive evaluation of fibrosis, pigmentation and dryness, skin condition was classified into 4 stages: Poor, Fair, Good, and Excellent. Fibrosis and pigmentation were judged based on physical findings, and dryness was judged based on the difference in skin moisture.

Measurement of urinary 8-hydroxy-2'-deoxyguanosine (8-OHdG). Midstream urine was used when needed as a sample for the measurement of urinary $8-\mathrm{OHdG}$. Immediately after collection, 100 $\mu \mathrm{l}$ of the urine sample was mixed with an equal volume of pure water (deionized water) and centrifuged. Sample urinary 8-OHdG was measured using a urinary oxidative stress-marker measurement system reader named ICR-001 (Techno Medica Co., Ltd., Yokohama, Kanagawa, Japan) based on the principle of immunochromatography (the value was corrected by the creatinine value obtained using the Jaffe method).

Ethical considerations. This study was conducted with the approval of the Graduate School of Health Sciences, Hirosaki University (2014-001), Mutsu General Hospital (H27-4) and Kagome Ethics Committee (2014-R06). The study was carried out after obtaining written informed consent from each patient. Kagome provided TJ used in this study. Kagome measured the serum carotenoid concentration under blind conditions. The focus of this study was lycopene and not the promotion of any product. Also, the researchers have not benefited from Kagome based on the research results. 
Table II. Skin surface temperature.

\begin{tabular}{lccccccccc}
\hline Term & Baseline & $2 \mathrm{w}$ & $3 \mathrm{w}$ & $4 \mathrm{w}$ & End & $1 \mathrm{M}$ & $3 \mathrm{M}$ & $6 \mathrm{M}$ & $12 \mathrm{M}$ \\
\hline Cases & 23 & 22 & 22 & 23 & 23 & 23 & 22 & 23 & 22 \\
Ipsilateral breast $\left({ }^{\circ} \mathrm{C}\right)$ & $34.6 \pm 1.5$ & $34.9 \pm 1.1$ & $34.8 \pm 1.1$ & $34.4 \pm 1.0$ & $35.0 \pm 1.5$ & $34.4 \pm 1.4$ & $34.7 \pm 1.1$ & $34.1 \pm 1.0$ & $33.3 \pm 0.9$ \\
Contralateral breast $\left({ }^{\circ} \mathrm{C}\right)$ & $34.1 \pm 1.8$ & $34.2 \pm 1.7$ & $33.8 \pm 1.5$ & $33.3 \pm 1.4$ & $34.0 \pm 1.8$ & $33.6 \pm 1.5$ & $33.5 \pm 1.5$ & $32.9 \pm 1.3$ & $32.8 \pm 1.1$ \\
dTemp & $0.48 \pm 0.62$ & $0.78 \pm 0.80$ & $1.01 \pm 0.70$ & $1.11 \pm 0.72$ & $0.97 \pm 0.81$ & $0.86 \pm 0.81$ & $1.19 \pm 0.89$ & $1.28 \pm 1.05$ & $0.43 \pm 0.91$ \\
$p$-Value & $p<0.01$ & $p<0.01$ & $p<0.01$ & $p<0.01$ & $p<0.01$ & $p<0.01$ & $p<0.01$ & $p<0.01$ & $p<0.05$ \\
$\quad$ Ipsilateral breast $v s$. & & & & & & & & &
\end{tabular}

Contralateral breast)

The skin surface temperature of ipsilateral beast in all measuring time points was significantly higher than that of the contralateral breast by Wilcoxon test. The values of dTemp, showing differences of skin surface temperature between ipsilateral and contralateral breasts, in $3 \mathrm{w}, 4 \mathrm{w}$, End, $3 \mathrm{M}$ and $6 \mathrm{M}$ were significantly higher than that in the Baseline by Mann-Whitney $U$-test $(p<0.05)$.

Statistical analysis. The measured data were analyzed after verifying normality using the Shapiro-Wilk test. Because of the nonparametric data, changes in serum carotenoid concentrations over time were compared for Baseline, End, $1 \mathrm{M}, 3 \mathrm{M}$ and $6 \mathrm{M}$ using the Kruskal-Wallis test. When there was a significant difference, the Mann-Whitney $U$-test was used for subsequent multiple comparisons. Furthermore, $p$-values were corrected using Shaffer's method. Urinary 8-OHdG value in urine was compared for Baseline, End, $1 \mathrm{M}, 3 \mathrm{M}$ and $6 \mathrm{M}$ using the Kruskal-Wallis test. The relationship between urinary $8-\mathrm{OHdG}$ value and serum lycopene concentration was first assessed for normality. Pearson's correlation analysis was performed if it showed normality, while Spearman's rank correlation analysis was performed if it did not. The comparison between the irradiation side and the non-irradiation side for skin surface temperature and skin moisture was performed using the Wilcoxon test. For dTemp, each point in time from the Baseline and $2 \mathrm{w}$ to $12 \mathrm{M}$ were compared using the Mann-Whitney $U$-test. For $\mathrm{dMoist}$, each point in time from the Baseline and $2 \mathrm{w}$ to $12 \mathrm{M}$ were compared with $t$-test. A chi-square test was performed to determine the relationship between the number of weeks of radiotherapy and skin reaction at the early stage, and the relationship between the skin reaction at the late stage for $6 \mathrm{M}$ and $12 \mathrm{M}$. The result is indicated as mean value \pm standard deviation. The analysis was performed using SPSS Statistics version 25 (IBM Co., Armonk, NY, USA). In each case, the significance level was set to less than $5 \%$.

\section{Results}

Serum carotenoid concentration. The total carotenoids concentration (total value of lutein, zeaxanthin, $\beta$-cryptoxanthin, $\alpha$-carotene, $\beta$-carotene and lycopene) was $2.36 \pm 0.99 \mu \mathrm{mol} / 1$ for Baseline, $2.19 \pm 1.09 \mu \mathrm{mol} / \mathrm{l}$ for End, $2.92 \pm 1.19 \mu \mathrm{mol} / 1$ for $1 \mathrm{M}, 2.97 \pm 1.12 \mu \mathrm{mol} / 1$ for $3 \mathrm{M}$ and $2.90 \pm 1.37 \mu \mathrm{mol} / 1$ for $6 \mathrm{M}$. The result of comparing Baseline and End with $1 \mathrm{M}, 3 \mathrm{M}$ and $6 \mathrm{M}$ showed an upward trend after $1 \mathrm{M}$, but there was no significant change over time (Figure 1, Kruskal-Wallis test, the $p$-value was corrected using the Shaffer method after the Mann-Whitney $U$-test).

Serum lycopene concentration. The lycopene concentration was $0.39 \pm 0.23 \mu \mathrm{mol} / \mathrm{l}$ at Baseline, $0.27 \pm 0.19 \mu \mathrm{mol} / \mathrm{l}$ at End,

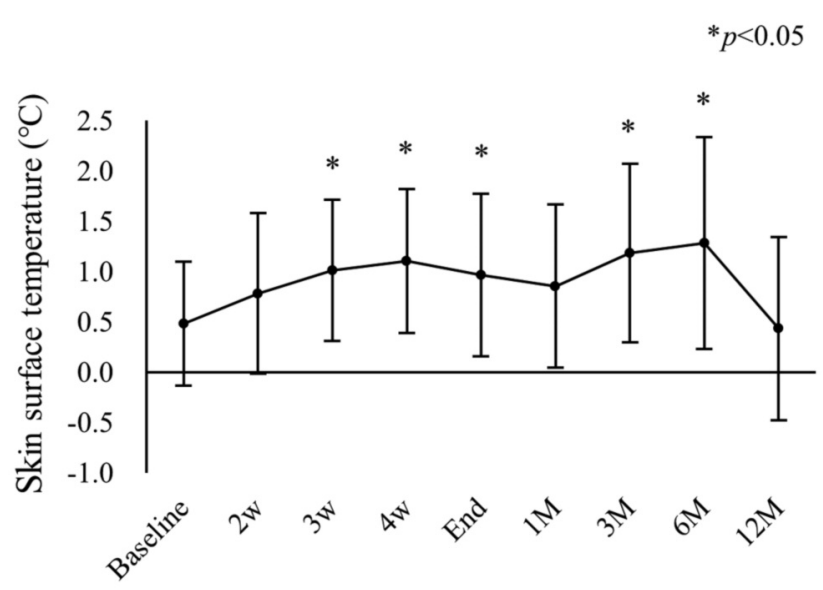

Figure 3. Serial changes in difference of skin surface temperature between ipsilateral and contralateral breasts (dTemp). The values of dTemp in $3 w, 4 w$, End, $3 M$ and $6 M$ were significantly higher than that in the Baseline by Mann-Whitney U-test $(p<0.05)$. Closed circle and bar show average and standard deviation, respectively.

$0.65 \pm 0.28 \mu \mathrm{mol} / 1$ at $1 \mathrm{M}, 0.78 \pm 0.34 \mu \mathrm{mol} / \mathrm{l}$ at $3 \mathrm{M}$, and $0.80 \pm 0.35 \mu \mathrm{mol} / 1$ at $6 \mathrm{M}$. The lycopene concentration at $1 \mathrm{M}$, $3 \mathrm{M}$ and $6 \mathrm{M}$ significantly increased compared to Baseline and End (Figure 2, $p<0.01$, Kruskal-Wallis test, $p$-value corrected using the Shaffer method after Mann-Whitney $U$-test).

\section{Skin condition}

Skin surface temperature. There was a significant difference in skin surface temperature between the irradiated and nonirradiated side at all times, from Baseline to $12 \mathrm{M}(p<0.05$, respectively, Wilcoxon test). The Baseline dTemp, which is the difference in skin surface temperature obtained by subtracting the value of the non-irradiated from the irradiated side, was compared with the dTemp obtained at other times. The results showed that dTemp at $3 \mathrm{w}, 4 \mathrm{w}$, End, $3 \mathrm{M}$ and $6 \mathrm{M}$ was significantly higher than at Baseline $\quad(p<0.05$ 
Table III. Skin moisture.

\begin{tabular}{|c|c|c|c|c|c|c|c|c|c|}
\hline Term & Baseline & $2 \mathrm{w}$ & $3 \mathrm{w}$ & $4 w$ & End & $1 \mathrm{M}$ & $3 \mathrm{M}$ & $6 \mathrm{M}$ & $12 \mathrm{M}$ \\
\hline Cases & 22 & 22 & 22 & 23 & 23 & 23 & 23 & 23 & 22 \\
\hline Ipsilateral breast (a.u) & $45.7 \pm 9.9$ & $37.6 \pm 14.7$ & $33.3 \pm 14.3$ & $25.0 \pm 10.8$ & $20.8 \pm 10.5$ & $30.9 \pm 16.4$ & $37.5 \pm 12.0$ & $41.9 \pm 13.1$ & $35.1 \pm 9.5$ \\
\hline Contralateral breast (a.u) & $44.9 \pm 10.9$ & $44.1 \pm 10.7$ & $39.7 \pm 12.2$ & $35.6 \pm 12.9$ & $37.0 \pm 11.5$ & $43.3 \pm 13.8$ & $45.9 \pm 11.4$ & $52.5 \pm 15.7$ & $40.6 \pm 9.1$ \\
\hline dMoist & $-0.35 \pm 8.63$ & $-6.44 \pm 8.06$ & $-6.36 \pm 8.20$ & $-10.55 \pm 8.57$ & $-16.25 \pm 8.04$ & $-12.33 \pm 15.78$ & $-8.36 \pm 8.89$ & $-10.54 \pm 10.37$ & $-5.50 \pm 7.50$ \\
\hline $\begin{array}{l}p \text {-Value } \\
\text { (Ipsilateral breast vs. } \\
\text { Contralateral breast) }\end{array}$ & n.s & $p<0.01$ & $p<0.01$ & $p<0.01$ & $p<0.01$ & $p<0.01$ & $p<0.01$ & $p<0.01$ & $p<0.01$ \\
\hline
\end{tabular}

The skin moisture of the ipsilateral breast in all timepoints except Baseline was significantly lower than that of the contralateral breast by Wilcoxon test. The values of dMoist, showing differences of skin moist between ipsilateral and contralateral breasts, in all measuring timepoints except Baseline were significantly higher than that in the Baseline by $t$-test $(p<0.05)$.

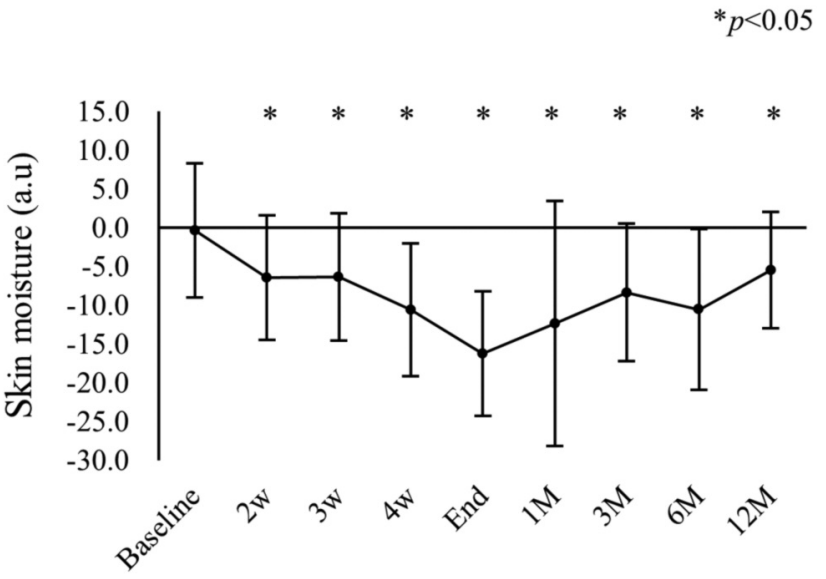

Figure 4. Serial changes in difference of skin moisture between ipsilateral and contralateral breasts (dMoist). The values of dMoist in all measured points except Baseline were significantly higher than that in the Baseline by t-test $(p<0.05)$. Closed circle and bar show average and standard deviation, respectively.

respectively). We also verified that dTemp was the highest at $6 \mathrm{M}$ and returned to the same level as Baseline at $12 \mathrm{M}$ (Table II, Figure 3, Mann-Whitney $U$-test).

Skin moisture. There was a significant difference in skin moisture between the irradiated and non-irradiated side at all times from $2 \mathrm{w}$ to $12 \mathrm{M}(p<0.01$, respectively, Wilcoxon test). The Baseline dMoist, which is the difference in skin moisture obtained by subtracting the value of the non-irradiated from the irradiated side, was compared with the dMoist values obtained at other times. As a result, dMoist was significantly higher than at Baseline at all times from $2 \mathrm{w}$ until 12M $(p<0.05$ respectively). Also, dMoist was the highest at End and gradually reduced towards $12 \mathrm{M}$ (Table III, Figure $4, t$-test).

Evaluation of skin adverse events. Radiodermatitis, which is an adverse event of the skin that occurs at the early stage,
Table IV. The number of patients for each Grade (CTCAE v4.0).

\begin{tabular}{lrrrrrc}
\hline & Baseline & $2 \mathrm{w}$ & $3 \mathrm{w}$ & $4 \mathrm{w}$ & End & $1 \mathrm{M}$ \\
\hline Grade0 & 23 & 23 & 22 & 18 & 1 & 23 \\
Grade1 & 0 & 0 & 1 & 5 & $22^{\mathrm{a}}$ & $0^{\mathrm{b}}$
\end{tabular}

Evaluation of serial changes of grade in radiation dermatitis as an early adverse event according to the Common Terminology Criteria for Adverse Events (CTCAE) v4.0. In the End timepoint, radiation dermatitis in most patients (22/23) was judged as Grade 1. However, for all the patients in $1 \mathrm{M}$, radiation dermatitis was judged as Grade 0 . aSignificant differences between End and Baseline $(p<0.01)$ by chisquare test. bSignificant difference between $1 \mathrm{M}$ and End $(p<0.01)$ by chi-square test; ${ }^{\mathrm{a}, \mathrm{b}} p<0.01$.

was evaluated using CTCAE (9). Though all subjects were grade 0 at Baseline, 1 subject was grade 0 and 22 subjects were grade 1 at the End timepoint. The number of subjects with grade 1 significantly increased at End (End: $p<0.01$, Table IV, Chi-square test). After that, at $1 \mathrm{M}$, all subjects were grade 0 and decreased significantly (1M: $p<0.01$, Table IV, Chi-square test). Skin adverse events that occur at later stages were evaluated using the EORTC scoring system (10). At $6 \mathrm{M}, 12$ subjects were excellent, 9 subjects were good and 2 subjects were fair. At $12 \mathrm{M}, 14$ subjects were excellent, 7 subjects were good and 2 subjects were fair (Figure 5).

Urinary 8-OHdG. Urinary 8-OHdG concentration was $26.8 \pm 12.5 \mathrm{ng} / \mathrm{mg} \mathrm{CRE}$ at Baseline, $26.0 \pm 8.5 \mathrm{ng} / \mathrm{mg} \mathrm{CRE}$ at End, $31.8 \pm 18.3 \mathrm{ng} / \mathrm{mg} \mathrm{CRE}$ at $1 \mathrm{M}, 29.1 \pm 13.2 \mathrm{ng} / \mathrm{mg} \mathrm{CRE}$ at $3 \mathrm{M}$ and $22.3 \pm 11.6 \mathrm{ng} / \mathrm{mg} \mathrm{CRE}$ at $6 \mathrm{M}$. $6 \mathrm{M}$ showed the lowest value, but no significant changes were observed (Figure 6, Kruskal-Wallis test).

Correlation between serum lycopene concentration and urinary $8-O H d G$. When the correlation between urinary 8 $\mathrm{OHdG}$ value and serum lycopene concentration was verified at End and 3M, a negative correlation was confirmed 

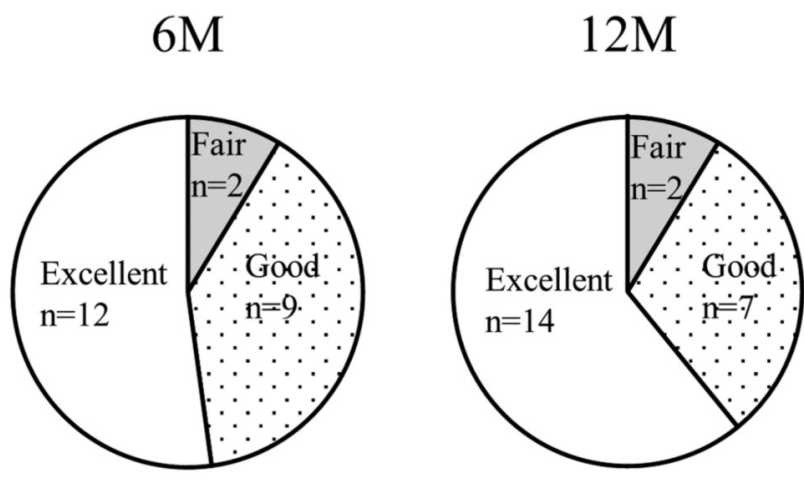

Figure 5. Evaluation of late adverse events of irradiated skin by the scoring system of European Organization for Research and Treatment of Cancer Global Cosmetic (EORTC). Most patients were judged as Good or Excellent in $6 \mathrm{M}$ and $12 \mathrm{M}$, although better in the latter.

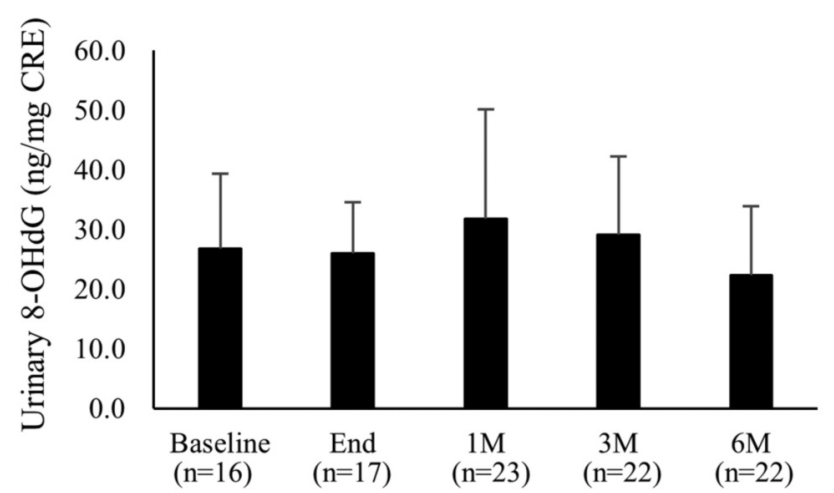

Figure 6. Serial changes in urinary 8-OHdG levels. There were no significant differences in the values. Column and bar show average and standard deviation, respectively.

respectively (Figure 7: End, Spearman's correlation analysis, correlation coefficient $-0.73, p<0.01$, Figure 8: $3 \mathrm{M}$, Pearson's correlation analysis, correlation coefficient -0.54 , $p<0.01)$.

\section{Discussion}

We examined in detail the effect of consuming tomato juice on the skin condition in the irradiation field after radiotherapy and its clinical usefulness in breast cancer patients following breast-conserving surgery. We found that a significant increase in serum lycopene concentration was observed at $1 \mathrm{M}$ from the start of $\mathrm{TJ}$ consumption. After that, the serum lycopene concentration remained at a high value even at $3 \mathrm{M}$ and $6 \mathrm{M}$. Concerning skin condition, high values were observed for dTemp and dMoist, showing the respective difference between the irradiated and non-

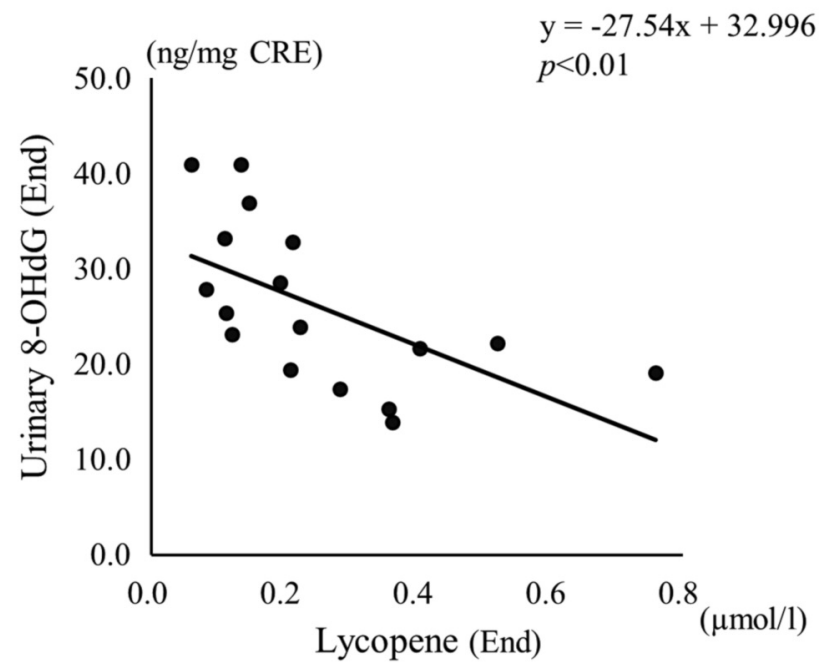

Figure 7. Relationship between concentration of lycopene and urinary 8-OHdG level in the End timepoint $(n=17)$. The correlation coefficient was -0.73 , indicating a negative correlation by Spearman's rank correlation coefficient $(p<0.01)$.

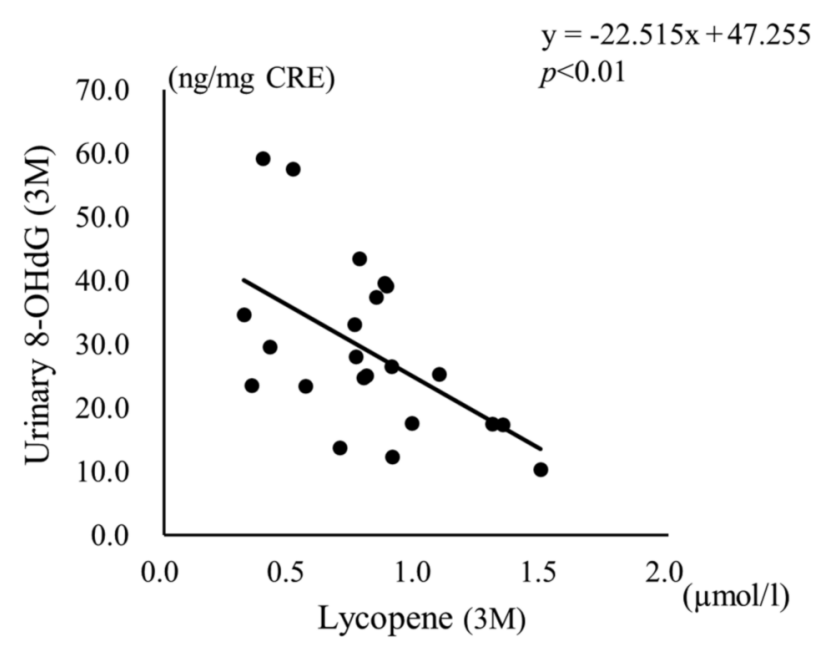

Figure 8. Relationship between concentration of lycopene and urinary 8-OHdG level in the $3 \mathrm{M}$ timepoint $(n=22)$. The correlation coefficient was -0.54 , indicating a negative correlation by Pearson's correlation coefficient $(p<0.01)$.

irradiated sides, after radiotherapy (dTemp: 6M, dMoist: End). However, at $12 \mathrm{M}$, the values returned to the level as before the start of irradiation. Also, recovery from the acute phase reaction of radiodermatitis was verified at $1 \mathrm{M}$.

According to a previous study by Sekine et al., dTemp for patients 12 months after radiotherapy was $1{ }^{\circ} \mathrm{C}$ or higher, and dTemp finally dropped to below $1^{\circ} \mathrm{C}$ after 24 months (12). In the current study, dTemp peaked at $6 \mathrm{M}$ with $\mathrm{TJ}$ 
consumption and was above $1^{\circ} \mathrm{C}$. However, we verified that dTemp was about $0.5^{\circ} \mathrm{C}$ at $12 \mathrm{M}$. Compared to previous studies, this indicated that dTemp decreased at an earlier stage. Concerning adverse events, these were categorized as Good and Excellent in approximately $90 \%$ of patients. In a previous study examining the late-stage adverse events in breast cancer patients for more than 3 years after radiotherapy (13), about $70 \%$ of the patients were reported to have been evaluated as Good and Excellent. These results show that the reduction of late adverse events confirmed in the current study is better than the previous study (13).

Among carotenoids, lycopene, an antioxidant contained in $\mathrm{TJ}$, has a particularly potent ability to eliminate singlet oxygen, which is a type of ROS. Food processing causes the mechanical breakdown of the food matrix and the release of lycopene. Lycopene taken into the digestive system is emulsified by ingested lipid and bile. The lycopene is solubilized into micelles by digestive enzymes and absorbed into the intestinal mucosa. Morever, the lycopene is taken into chylomicron and secreted into the lymphatic system for transport to the liver and peripheral tissues (14). Absorbed lycopene is known to accumulate specifically in testis in males and also to be distributed in breast and skin (15).

As for lycopene concentration in the skin, Scarmo et al. have reported that serum lycopene concentration has a direct correlation with lycopene concentration in the skin (16). Ribaya-Mercado et al. have stated that lycopene accumulated in the skin reduces oxidative damage in tissues caused by UV irradiation (17). In addition, it has been reported that lycopene can protect against cell damage that is caused by ionizing radiation with in vitro and animal studies (3).

Radiotherapy causes DNA damage to cancer cells in the irradiated tissue, causing damage not only to the skin but also to deeper mammary, adipose and muscle tissue (18). The reports described above $(3,16,17)$ indicate that consumption of TJ may increase the lycopene concentration in the body and could promote recovery of damage to and inflammation of irradiated tissues, including the skin. The recovery of skin condition at the early stage shown in this study is due to the increase in lycopene concentration in the body by TJ consumption, suggesting that the deterioration and prolongation of dermatitis may have been inhibited through the elimination of ROS produced in the skin and inhibition of DNA oxidative damage. However, we were not able to verify the relationship between serum carotenoid concentration and skin condition. Also, the direct action of serum lycopene concentration on the skin condition was not explicit. The relationship between serum lycopene concentration and skin condition could not be clarified in this study. One of the reasons could be that the degree of radiodermatitis is affected by individual differences, including radiotherapy dose-volume of breast. Since it is not possible to restrict the intake of food containing antioxidants other than TJ in clinical practice, it is presumed that various exogenous antioxidants other than carotenoids might have had an effect.

For examining the relationship between TJ and 8-OHdG, Nakamura et al. (5) conducted a study in humans by irradiating blood with different doses of X-rays at 3 different time points, before and after consumption of TJ, and after washout to evaluate DNA damage of lymphocytes and condition of oxidative stress in serum $8-\mathrm{OHdG}$. They stated that in individuals with low lycopene and $\beta$-carotene concentration prior to TJ consumption, the serum levels of 8-OHdG and radiation-induced dicentrics after consuming TJ were significantly reduced compared to those before consumption. In the current study, the values of urinary 8$\mathrm{OHdG}$ were lower in individuals with higher serum lycopene concentration at the end of radiotherapy. Based on this, increasing the serum carotenoid concentration after completion of radiotherapy could lead to recovery from damage to cells caused by radiation treatment and improvement of oxidative stress conditions. Tanaka et al. examined the effect of the continuous intake of mixed juice of vegetables and fruits containing $\beta$-carotene and $\alpha$-carotene on the skin condition of healthy human volunteers (19). They reported an improvement of skin elasticity and texture. In line with this, an increase in serum carotenoid concentration can have a positive effect on the skin condition that is seen not only in early adverse events but also in late adverse events. Therefore, consumption of TJ after radiotherapy was considered significant for the recovery of irradiated tissues and the improvement of oxidative stress. In addition, although we focused on lycopene in the current study, TJ contains various carotenoids other than lycopene, which may have affected the skin condition to a certain extent. Further investigation should be performed concerning antioxidants.

Finally, the suitability of $8-\mathrm{OHdG}$ (20), which was selected as a marker to reflect the effects of oxidative stress in organisms, is described below. $8-\mathrm{OHdG}$ is a DNA oxidative stress marker that is susceptible to oxidative stress induced by ROS (20). Also, we have confirmed that 8-OHdG is a marker associated with inflammation levels from the correlation between $8-\mathrm{OHdG}$ and variations in matrix metalloproteinase (MMP) associated with exercise load (21). From this, it was expected that changes in oxidative stress conditions in the body would be reflected. However, in the current study, no significant changes were observed before and after radiotherapy, and there was no apparent effect for both radiotherapy and TJ consumption. The cause of this was considered to be due to various kinds of stressors, in addition to the damage caused by irradiation to the subjects of this study before and after radiotherapy. For example, the longterm effects of surgery and cancer chemotherapy, as well as the psychological stress experienced by the patients during cancer treatment and follow-up can be stressors that are 
equal to or exceed that of radiotherapy. It is considered that the reduction of oxidative stress by TJ consumption in this study may have been masked or modified by these stressors (22). We want to conduct further studies in the future for the oxidative stress markers by including the selection of markers with different targets such as intracellular lipids.

\section{Conclusion}

Long-term consumption of tomato juice increases serum carotenoid concentration and contributes to the recovery of early and late skin adverse events caused by radiotherapy. However, in this study, we were unable to ascertain the details of the mechanism by which carotenoids reduce damage to the irradiated tissues. Further studies will be required to clarify this.

\section{Conflicts of Interest}

The Authors have no potential conflicts of interest to disclose.

\section{Authors' Contributions}

YM and CI designed the research. Kyogo Y and YM examined, treated and diagnosed subjects. YF, Kazue Y, AS and CI conducted the research. Hitoshi $\mathrm{S}$ and $\mathrm{AH}$ proceeded and analyzed the data. YF performed the statistical analyses and AH, SS, KA, Hiroyuki S, YM and CI interpreted the data. YF, AH, SS, KA, Hiroyuki S, YM and CI wrote the paper. YM had primary responsibility for the final content. All Authors contributed to and approved the final version of the manuscript.

\section{Acknowledgements}

This work was supported by JSPS KAKENHI Grant Numbers JP26670950 (C.I.) and JP25461900 (Y.M.). The Authors would like to thank all subjects who participated in this project.

\section{References}

1 Borek C: Antioxidants and radiation therapy. J Nutr 134(11): 3207S-3209S, 2004. PMID: 15514309. DOI: 10.1093/jn/134. $11.3207 \mathrm{~S}$

2 Cicero AFG and Colletti A: Effects of carotenoids on health: Are all the same? Results from clinical trials. Curr Pharm Des 23(17): 2422-2427, 2017. PMID: 28176669. DOI: $10.2174 / 1381612823666170207095459$

3 Gajowik A and Dobrzyńska MM: Lycopene - antioxidant with radioprotective and anticancer properties. A review. Rocz Panstw Zakl Hig 65(4): 263-271, 2014. PMID: 25526570.

4 Naito Y, Lee M, Kato Y, Nagai R and Yonei Y: Oxidative stress markers. Anti-Aging Medicine 7(5): 36-44, 2010 (In Japanese).

5 Nakamura A, Itaki C, Saito A, Yonezawa T, Aizawa K, Hirai A, Suganuma H, Miura T, Mariya Y and Haghdoost S: Possible benefits of tomato juice consumption: a pilot study on irradiated human lymphocytes from healthy donors. Nutr J 16(1): 27, 2017. PMID: 28494764. DOI: 10.1186/s12937-017-0248-3
6 Singh M, Alavi A, Wong R, and Akita S: Radiodermatitis: A review of our current understanding. Am J Clin Dermatol 17(3): 277-292, 2016. PMID: 27021652. DOI: 10.1007/s40257-016$0186-4$

7 Harms-Ringdahl M, Jenssen D and Haghdoost S: Tomato juice intake suppressed serum concentration of 8-oxodG after extensive physical activity. Nutr J 11: 29, 2012. PMID: 22551119. DOI: $10.1186 / 1475-2891-11-29$

8 Yamamoto Y, Aizawa K, Mieno M, Karamatsu M, Hirano Y, Furui K, Miyashita T, Yamazaki K, Inakuma T, Sato I, Suganuma $\mathrm{H}$ and Iwamoto T: The effects of tomato juice on male infertility. Asia Pac J Clin Nutr 26(1): 65-71, 2017. PMID: 28049263. DOI: 10.6133/apjen.102015.17

9 Common Terminology Criteria for Adverse Events (CTCAE) version 4.0-JCOG. Available at: http://www.jcog.jp/doctor/tool/ ctcaev4.html [Last accessed on 6th April 2019]

10 Winchester DP and Cox JD: Standards for breast-conservation treatment. CA Cancer J Clin 42(3): 134-162, 1992. PMID: 1568135. DOI: $10.3322 /$ canjclin.42.3.134

11 Kitajima M, Mikami K, Noto Y, Itaki C, Fukushi Y, Hirota Y, Mariya Y, Tsushima M, Kattou K and Osanai T: Quantitative assessment of radiodermatitis through a non-invasive objective procedure in patients with breast cancer. Mol Clin Oncol 12(1): 89-93, 2020. PMID: 31814981. DOI: 10.3892/mco.2019.1948

12 Sekine H, Kobayashi M, Honda C, Aoki M, Nakagawa M and Kanehira C: Skin reactions after breast-conserving therapy and prediction of late complications using physiological functions. Breast Cancer 7(2): 142-148, 2000. PMID: 11029786. DOI: 10.1007/bf02967446

13 Nozaki M, Kagami Y, Mitsumori M and Hiraoka M: A multicenter investigation of late adverse events in Japanese women treated with breast-conserving surgery plus conventional fractionated whole-breast radiation therapy. Jpn J Clin Oncol 42(6): 522-527, 2012. PMID: 22504781. DOI: 10.1093/jjco/hys050

14 Wang XD: Lycopene metabolism and its biological significance. Am J Clin Nutr 96(5): 1214S-1222S, 2012. PMID: 23053559. DOI: $10.3945 /$ ajen.111.032359

15 Kong KW, Khoo HE, Prasad KN, Ismail A, Tan CP and Rajab NF: Revealing the power of the natural red pigment lycopene. Molecules 15(2): 959-987, 2010. PMID: 20335956. DOI: $10.3390 /$ molecules 15020959

16 Scarmo S, Cartmel B, Lin H, Leffell DJ, Welch E, Bhosale P, Bernstein PS and Mayne ST: Significant correlations of dermal total carotenoids and dermal lycopene with their respective plasma levels in healthy adults. Arch Biochem Biophys 504(1): 34-39, 2010. PMID: 20637178. DOI: 10.1016/j.abb.2010.07.004

17 Ribaya-Mercado JD, Garmyn M, Gilchrest BA and Russell RM: Skin lycopene is destroyed preferentially over beta-carotene during ultraviolet irradiation in humans. J Nutr 125(7): 18541859, 1995. PMID: 7616301. DOI: 10.1093/jn/125.7.1854

18 Goyal S, Buchholz TA and Haffty BG: Breast Cancer: Early Stage. In: Perez and Brady's Principles and Practice of Radiation Oncology Sixth Edition. Halperin EC, Wazer DE, Perez CA, Brady LW (eds.) Philadelphia, PA, Lippincott Williams \& Wilkins, pp. 1107-1114, 2013.

19 Tanaka S, Okame T, Ohkawara A, Sunabori S and Suganuma H: Effects of continual intake of mixed vegetable and fruit juice on skin condition. Pharmacometrics 90(1/2): 13-24, 2016 (In Japanese). 
20 Valavanidis A, Vlachogianni T and Fiotakis C: 8-hydroxy-2'deoxyguanosine (8-OHdG): A critical biomarker of oxidative stress and carcinogenesis. J Environ Sci Health C Environ Carcinog Ecotoxicol Rev 27(2): 120-139, 2009. PMID: 19412858. DOI: $10.1080 / 10590500902885684$

21 Fukushi Y, Nakamura A, Shiroto Y, Tanaka K, Ito T, Nozawa Y, Itaki $\mathrm{C}$ and Mariya Y: Serial changes of oxidative stress marker 8-hydroxy-2'-deoxyguanosine and inflammation-related markers matrix metalloproteinases induced by exercise load. A usability study on serum, saliva and urine samples. J Health Sci Res 7: 13-20, 2017 (In Japanese).
22 Fukushi Y, Nakamura A, Itaki C, Tokonami S, Yamada M and Mariya Y: Mental and physical stress of the Fukushima disaster evacuees as estimated by the measurement of urinary 8-hydroxy2'-deoxyguanosine. Exp Ther Med 16(1): 231-235, 2018. PMID: 29896244. DOI: 10.3892/etm.2018.6165

Received May 22, 2020

Revised June 5, 2020

Accepted June 15, 2020 